\title{
INFORMACIÓN Y MANIPULACIÓN: ¿CÓMO PROTEGER LOS SERES VIVOS VULNERADOS? LA PROPUESTA DE LA BIOÉTICA DE LA PROTECCIÓN
}

\author{
Fermin Roland Schramm \\ Escola Nacional de Saúde Pública ENSP/FIOCRUZ, Rio de Janeiro, Brasil. \\ roland@ensp.fiocruz.br
}

\begin{abstract}
Resumen: Muchas de las cuestiones abordadas por la ética aplicada y la bioética atañen al contexto discursivo de las prácticas de comunicación y de cómo es utilizada la información, pues del contexto en los cuales se dan los actos lingüísticos pueden depender la fuerza argumentativa para la solución de un conflicto moral entre actores sociales con intereses y valores diferentes. Considerando que todo acto comunicativo tiene una dimensión denotativa (que se refiere a aquello que el mensaje pretende decir) y una connotativa (referente a aquello que se añade al mensaje debido a como el código es utilizado o a su "retórica"), y que, dependiendo de las circunstancias (dadas por el contexto y la situación de tal acto), la connotación puede ser el medio en cual se dan las formas de abuso de poder como las varias formas de manipulación de la información, surge la necesidad de dar amparo a los sujetos vulnerados por tales prácticas. Nuestra propuesta es intentar tal operación con las herramientas de una vertiente de la bioética de la protección, que llamaremos Bioética de la Protección lato sensu, para distinguirla de su vertiente stricto sensu.
\end{abstract}

Palabras-clave: Mensaje. Denotación. Connotación. Información. Manipulación. Bioética de la Protección.

Abstract: The questions approached by applied ethics and bioethics correspond to the discursive context of communication practices and of how information is used, for the context in which the linguistic acts occur may depend on the argumentative force (or cogency) for the solution of a moral conflict between social actors with different interests and values. Considering that every communicative act has a denotative dimension (which refers to what the message intends to say) and a connotative one (referring to what is added to the message because of the way the code is used, or its "rhetoric"), and that, depending on the circumstances (given by the context and the situation of such act), the connotation may be the environment in which forms of power abuse may take place as the different ways to manipulate information, and where the need to give support to the subjects vulnerable to these practices may arise. Our proposal is to try such operation with the instruments of protection bioethics line we will call Bioethics of Protection lato sensu, to differentiate it from the strictu sensu line.

Key words: Message. Denotation. Connotation. Information. Manipulation. Bioethics of Protection lato sensu. 
M uchas de las cuestiones abordadas por la ética aplicada y, en particular, por la bioética, atañen a la calidad de la información que circula entre los actores sociales y al modo por el cual la información es tratada en las prácticas de comunicación, puesto que de la calidad de la información y del tipo de interrelación puede depender el tipo de solución que se intenta dar a un conflicto moral.

Por supuesto, y de acuerdo con la semiótica contemporánea, en las prácticas comunicativas siempre existe una parte denotativa, referente a aquello que el mensaje de un emisor efectivamente dice - o pretende decir - al destinatario; y una parte connotativa, que puede ser entendida como aquello que, en determinadas circunstancias, el mensaje puede adicionalmente indicar según cómo el código es utilizado; siendo posible, por ende, hablar de la dupla dimensión de cualquier acto comunicativo.

Así, la manera cómo un mensaje es utilizado - y que se refiere específicamente a la dimensión connotativa del mensaje - hace parte del así llamado "arte de convencimiento" (que es la retórica), que siempre acompaña tal mensaje y, por lo tanto, siempre afecta a la práctica comunicativa como un todo. Es por eso que se puede hablar de manera pertinente de "manipulación" de la información, hecho éste que implica siempre una estructura de poder y puede, además, tener una estructura de dominación propiamente dicha, que afecta a las relaciones interpersonales y las interrelaciones sociales posibilitando, por lo tanto, que se perjudique a una parte de los actores involucrados en una acción comunicativa.

Si lo que decimos es correcto, surge también el problema, moralmente significativo, de cómo proteger a los eventuales afectados contra abusos de poder, o de dominación; en particular, contra eventuales daños infligidos a sujetos y poblaciones más frágiles o susceptibles. Existen, evidentemente, muchas maneras correctas para abordar este problema, empezando por los medios de autodefensa personales, pasando por los medios corporativos (cuando existen), y aquellos que tienen una pretensión universalista, como los jurídicos o los de las políticas públicas. Pero tales medios, aunque probablemente efectivos, no pertenecen propiamente a la esfera de la ética aplicada, aunque puedan tener interfaces con ella.

Por eso, propondremos aquí la utilización de la Bioética de la Protección, que consideramos una herramienta adecuada para dar cuenta de este tipo de conflictos en el campo de las acciones humanas, que involucran y pueden afectar de manera significativa e irreversible a otros humanos y, más en general, a otros seres vivos. 


\section{Información, Comunicación y Malentendidos}

La información, contrariamente a la comunicación, puede ser considerada como la parte del mensaje susceptible de manipulación; y eso de dos maneras. Primero porque ella es considerada, por regla, como meramente cuantitativa y objetivamente mensurable, por ejemplo, por una función logarítmica (como en la Teoría de la Información clásica de Claude Shannon). Sin embargo, el aspecto "objetivamente" mensurable es, por supuesto, solamente una de las características de la información - la que se refiere a la dimensión denotativa - siendo que subsisten también los aspectos cualitativos, que se refieren a la dimensión connotativa y que son de más difícil interpretación, debido a su polisemia estructural que permite una interpretación prácticamente infinita (BARTHES, 1973).

Pero lo más importante para nosotros es que los aspectos connotativos pueden relacionarse a las prácticas humanas que involucran conflictos de intereses y estructuras de poder y de dominación de unos humanos sobre los otros; y son esos aspectos cualitativos que, en nuestra opinión, más deben preocupar a los filósofos morales y los bioeticistas, pues tales aspectos pueden implicar daños efectivos resultantes de cómo la información es tratada y manipulada por unos en contra de otros. Ése puede ser el caso del tipo de información suministrada a los involucrados en investigaciones científicas (con finalidades clínicas o no) por investigadores y sus patrocinantes, pero puede ser también aquél de la calidad de la información dada por medios de comunicación sobre asuntos polémicos y que implican una fuerte carga emocional en el público, como la mayoría de los asuntos abordados por la bioética.

Así, se puede decir que la información tendría una tendencia a la manipulación por ser estructuralmente unidireccional (por eso llamada también de transmisión). Porque, en su propia dinámica retórica, es no dialógica, siendo posible, además, decir que la concepción que la considera objetiva y mensurable depende probablemente de esta su característica supuestamente tan sólo cuantitativa. Evidentemente, siempre se puede argumentar que el diálogo y la comunicación de hecho no existen, pues - por razones que solamente los psicoanalistas explicarían - en la comunicación efectiva el emisor recibe del destinatario su propio mensaje invertido (como pretendía Lacan), o que sólo existen "malentendidos" (como muestra El Extranjero de Albert Camus). O sea, que el diálogo - en el sentido platónico de "compartir el lógos" - de hecho no existiría. Debido a la propia estructura del deseo humano, que siempre sería una estructura de poder y que, por lo 
tanto, no permitiría al yo descentralizarse hacia el otro para compartir con él algo más que el goce del dominio o de la prevaricación; y que fuese capaz de instituir, en la estructura imaginaria de lo social, una relación de reconocimiento recíproca, hecha de un "tú" frente a un "yo", considerados ambos actores necesarios a la auténtica comunicación que, por definición, siempre implica una estructura por lo menos dual: un yo y un otro, un yo y un tú, un nosotros y los otros, nosotros y ustedes - una estructura capaz de compartir el lógos de la manera más amplia posible.

Sea como fuere, las implicaciones manipuladoras de la información, que pueden ser estructurales o contingentes, nos ponen el problema de cómo proteger los afectados contra eventuales daños evitables. Una de las maneras para pensarlo es la propuesta, hecha aquí, de una Bioética de la Protección.

\section{La Bioética de la Protección}

La expresión Bioética de la Protección se refiere a una vertiente reciente de la bioética, formulada inicialmente por Kottow y Schramm para intentar dar cuenta de los conflictos y dilemas morales enfrentados por la salud pública en América Latina; y que, en nuestra evaluación, no pueden ser resueltos concretamente por las demás herramientas de la bioética mundial; en particular, por el padrón norteamericano conocido como bioética principialista (SCHRAMM \& KOTTOW, 2001).

\section{Los dos sentidos de la Bioética de la Protección}

La Bioética de la Protección puede ser entendida de dos maneras distintas, aunque no excluyentes, y de acuerdo con el sentido, más o menos amplio, de la palabra protección: (a) stricto sensu y (b) lato sensu, siendo que el primer sentido debe ser comprendido como más específicamente técnico y el segundo como más general, puesto que el primero está probablemente incluido lógicamente en el segundo.

En el sentido estricto, la Bioética de la Protección se refiere específicamente a las medidas que deben, necesariamente, ser tomadas para proteger a individuos y poblaciones que no disponen de otras medidas que les garanticen las condiciones indispensables para llevar adelante una vida digna y no solamente disponer de una sobrevida o - de acuerdo con la terminología de 
Giorgio Agamben - de su "vida desnuda"; y que son, por lo tanto, excluidos de la "comunidad política" y de las políticas de los derechos humanos (AGAMBEN, 1996).

Ya en su sentido más general, la bioética de la protección tiene una ambición mayor. Pues, aunque continúe otorgando la prioridad léxica a los intereses de los más desamparados (para no perder su preocupación inicial con los medios necesarios para ofrecer contenido concreto al ideal de justicia social) y defendiendo, por ende, acciones de tipo afirmativo con una finalidad equitativa; tiende a pensarse teleológicamente - de acuerdo con la terminología defendida por Derrida - como la ética de una "nueva forma de cosmopolitismo" y de una "democracia futura" (démocratie à venir) fundada en una "hospitalidad incondicional", substraída a cualquier forma de cálculo y de manipulación. Es decir, "[una hospitalidad que se exponga], sin límites, a la venida del otro, más allá del derecho, más allá de la hospitalidad condicionada por el derecho de asilo, por el derecho a la inmigración, por la ciudadanía y mismo por el derecho a la hospitalidad universal de Kant, la cual permanece todavía controlada por un derecho político o cosmopolita, [pues] solamente una hospitalidad incondicional puede dar sentido y su racionalidad práctica a cualquier concepto de hospitalidad" (DERRIDA, 2003).

El sentido estricto de la Bioética de la Protección tiene una prioridad léxica sobre el sentido más general. Constituye una especie de "núcleo duro" de la propuesta (en el sentido de Lakátos), sin el cual la propuesta protectora se transformaría en más una "buena intención" de hecho ineficaz, sobretodo cuando se considera la situación de los grandes contingentes poblacionales del Tercer Mundo, quienes viven abajo de la línea de pobreza y sin el mínimo de asistencia; es decir, que viven en la mera condición de la "vida desnuda". En ese sentido, me parece que el análisis de Agamben es prima facie más pertinente para la situación de los países del Tercer Mundo. Sin embargo, no debemos olvidar los proyectos que intentan cambios radicales en esa situación de mundos separados, que es la característica de la actual "globalización excluyente".

En ese sentido, se puede muy pertinentemente argumentar que la sospecha de una creciente despolitización de los derechos humanos tiene sentido. Pues, en su versión actual defendida por muchos países del Primer Mundo (muchas veces siendo una especie de epifenómeno de la lucha contra el así llamado "terrorismo mundial"(ZIZEK, 2003), tales derechos están de hecho reducidos al mero "asistencialismo" sin, adicionalmente, mostrar una preocupación más sustancial con proyectos más amplios y efectivos de justicia social, que fuesen 
capaces de incluir a todos los sujetos amenazados en su calidad de salud y su propia vida desnuda, como objeto de su preocupación moral, lo que se parece, por otra parte, a la preocupación del proyecto derridiano de la hospitalidad incondicional. Dicho de otra manera, es prima facie correcto priorizar la forma de "humanitarismo [entendido como] pura defensa de los inocentes y los débiles contra el poder [y] como defensa prepolítica" de individuos y poblaciones en sus condiciones de la simple vida desnuda. Y se puede preguntar qué pasa, de hecho, con los derechos humanos cuando ellos son reducidos a los "derechos" de aquellos que son excluidos de la comunidad política y relegados a su condición de vida desnuda, visto que se trata de los derechos "de aquellos que, justamente, no tienen derechos, que son tratados como no humanos", y se puede también sospechar que "la política puramente humanitaria y antipolítica de apenas prevenir el sufrimiento equivale, en la práctica, a la prohibición implícita de elaborar un proyecto colectivo de transformación sociopolítica" (ZIZEK, 2004). Ésta es, en nuestra opinión, la principal razón para comprender a la bioética de la protección en su doble sentido.

\section{La Bioética de la Protección es, ante todo, una bioética}

Pero, la Bioética de la Protección es, ante todo, una bioética; es decir, una ética aplicada a las prácticas humanas en el mundo de la vida, comprendida ya sea como mera zoé (o "vida desnuda") o como bíos (o vida típicamente humana, que incluye, desde Aristóteles, la vida moral). Como tal, pretende ser una herramienta teórica y práctica capaz de dar cuenta de las prácticas humanas que involucren seres vivos y, en particular, seres humanos. O sea, una herramienta capaz de dar cuenta de la moralidad de tales prácticas, cuando ellas tienen, o pueden tener, efectos significativos irreversibles sobre los seres vivos afectados por tales prácticas (KOTTOW, 1995). Dicho de otra manera, como ética aplicada a las prácticas humanas que involucran seres vivos, la bioética puede ser considerada una herramienta para dar cuenta de como los seres humanos manejan los conflictos de intereses y de valores entre sí, e intentan resolverlos de manera pacífica y sin recurrir al orden jurídico. Y eso de dos maneras complementarias: (1) por el "análisis racional e imparcial" de tales conflictos, lo que constituye, por así decir, la condición formal necesaria para enfrentar los conflictos; y (2) por la prescripción de aquello que, en una situación determinada, debe ser hecho; y por la proscripción de aquello que, al contrario, debe ser evitado, o sea, 
por la preocupación normativa frente a los conflictos, lo que constituye, por así decir, condición suficiente para tal solución.

La Bioética de la Protección, ampliamente comprendida, intenta también rescatar el sentido, probablemente más antiguo en la cultura griega, de la palabra ethos (guarida), que es, justamente, el sentido que proponemos de protección. Con eso, no pretendemos evidentemente decir que el sentido más antiguo sea el más pertinente o el más correcto, pues el idioma y sus sentidos se transforman históricamente. Pero las argumentaciones anteriores sobre la "vida desnuda" y la "hospitalidad incondicional" parecen indicar que el sentido de protección es aún pertinente - y pendiente - en el campo de la ética aplicada hoy, al lado de los sentidos posteriores de "costumbres" sociales y de "hábitos" individuales. De hecho, si pensamos, sobretodo, en su sentido estricto de protección de la vida desnuda, de "guarida" para los desamparados y afectados, es innegable que él se aplica a las enormes masas de individuos sin ningún tipo de asistencia y a los cuales las políticas de los derechos humanos muy probablemente suenan como una mera abstracción. Dicho de otra manera, como cualquier otra ética de la vida (comprendida tanto en su sentido estrictamente biológico de zoé como en un sentido más antropológico de bíos), la Bioética de la Protección pretende inscribirse en el campo de las éticas aplicadas, las cuales "se aplican" a los conflictos morales (referentes a los "hábitos" interpersonales y sociales) que surgen en la praxis humana; y, además de eso, pretende también dar cuenta de situaciones "emergenciales", representadas por las vidas desnudas desamparadas y vulneradas.

\section{Aplicabilidad de la Bioética de la Protección}

Suele decirse que la bioética es una herramienta que se aplica a los conflictos de intereses y valores vigentes en las sociedades contemporáneas; que serían, en su mayoría, sociedades seculares y pluralistas, en las cuales existiría una pluralidad de valores supuestamente capaz de abarcar la totalidad de las construcciones imaginarias y simbólicas que instituyen y constituyen la convivencia humana democrática y, eventualmente, aquella entre los actores de la biosfera en su totalidad (de acuerdo con la propuesta de Derrida). Convivencia entendida, filológicamente, como un "vivir juntos" no sólo en el sentido de un sobrevivir biológico stricto sensu (de la zoé o de la vida desnuda), sino adicionalmente como el compartir de una humanidad común, en el sentido de permitir a cada miembro de la especie 
homo sapiens sapiens tener las condiciones necesarias e indispensables para no solamente sobrevivir como cualquier ser vivo no humano perteneciente a la zoé, sino, además, realizar sus proyectos de vida "razonables" o compatibles con los proyectos de los otros, próximos o distantes, que sean y que pertenecen al bíos típicamente humano, lo cual debe necesariamente incluir la dimensión ética. En suma, para que cualquier ser humano pueda tener una calidad de vida compatible con aquella de los demás seres humanos y, eventualmente, de los demás seres vivos (que no lo amenacen de manera directa en su "vida desnuda"). Eso parece estar de acuerdo con la intuición de Hans Jonas, según la cual una ética práctica (no reducida a una mera ética formal, una "metaética" o una "ciencia de la moral" abstracta), correctamente comprendida, debe necesariamente enfrentar un antiguo problema existencial y, por ende, también propiamente filosófico - iniciado explícitamente en el ámbito de la filosofía occidental por la discusión entre Parménides y Heráclito, y referente a la permanencia, o no, del Ser frente al No-Ser - y que, en la época contemporánea, se reformuló como dialéctica entre el Ser y la Nada gracias a las contribuciones sobre todo de Heidegger y de Sartre. Esta preocupación con la permanencia del Ser frente al Nada se refiere, en Jonas, principalmente a las generaciones futuras y a la calidad de sus vidas, que pueden ser afectadas sensiblemente por nuestras prácticas actuales. Pero la calidad de vida debe ser referida también a los seres humanos actuales, intentando superar la fractura existente entre las vidas desnudas y las vidas que pueden, razonablemente, ser llamadas "decentes".

Considerando que la bioética tiene por lo menos dos funciones reconocidas y distintas, pero no separadas: una específicamente teóricocrítica - o analítica -; la otra propiamente práctica - o normativa -; y considerando, además, que (a) la función normativa no siempre es efectiva (no siempre es aplicada en el sentido de tener efectos concretos en la solución de un conflicto: las normas pueden ser respetadas o no), y que (b) existen situaciones diferentes, y moralmente cuestionables porque "inicuas" para individuos, poblaciones, naciones y regiones en el así llamado proceso de globalización, se puede también inferir que las tradicionales herramientas "descriptiva" y "normativa" sean, de hecho, insuficientes en su vocación práctica. Siendo así, tenemos una buena razón para proponer una Bioética de la Protección, la cual pretende añadir a las dos funciones tradicionales de la bioética una tercera: (c) la herramienta protectora, la cual, como indica la propia palabra, visa proteger, o "dar amparo", a la propia vida humana (y no humana) para que ella tenga las posibilidades de continuar "siendo". 
De hecho, de acuerdo con Jonas, esa función de la ética es bien conocida por los padres y madres que protegen sus hijos hasta que puedan desarrollar medios propios para vivir sus vidas de relación responsable. Se puede, mutatis mutandis, pensar medidas protectoras sociales para que un Estado ética y socialmente legítimo proteja, con prioridad impostergable, a sus ciudadanos frente al desamparo de sus individuos y poblaciones que solamente tienen sus vidas desnudas.

\section{Conclusiones}

Pero, al final, ¿qué tiene que ver la Bioética de la Protección con la manipulación de la información? Como intentamos mostrar, la tercera función de la bioética - además de la descriptiva y normativa - es la función protectora, la cual puede ser entendida de dos maneras distintas, aunque no excluyentes: stricto sensu y lato sensu.

En el sentido estricto, o técnico, la protección tiene que ver con una condición necesaria para que se pueda decir, con alguna pertinencia, que la bioética es una herramienta capaz de permitir a cualquier ser humano tener una calidad de vida por lo menos razonable y de acuerdo con los así llamados derechos humanos fundamentales, que necesitan, para que no sean mero flatus vocis, que cualquier representante de la especie humana tenga las condiciones necesarias para poder vivir junto con sus semejantes a fin de realizar su proyecto de vida razonablemente compatible con los demás.

En el sentido lato, la Bioética de la Protección pretende dar "guarida" a todos los involucrados en algún conflicto de intereses, de acuerdo con el proyecto de la hospitalidad incondicional propuesto por Derrida.

En los dos casos, el acceso a la información es vital. Pero tal información debe ser la más libre y amplia posible; pues una información manipulada, aún cuando pretende subrepticiamente "proteger" el destinatario de verdades incómodas, de hecho añade al desamparo el sentimiento de impotencia, resultante de la información según la cual ilas cosas están cómo están pues ésta es su naturaleza!

La Bioética de la Protección no puede aceptar ese "estado de cosas" como se fuese natural. En este sentido, la práctica de la protección no implica necesariamente que el destinatario (que es un sistema evolutivo) esté desprovisto de alguna capacidad de comprender y decidir lo que puede ser mejor para él y para salir de su condición de mera "vida desnuda" para 
entrar en un mundo habitable para él y que Derrida define, muy correctamente, como un mundo de la hospitalidad incondicional.

Concluyendo, la protección no excluye a priori el ejercicio de la autonomía (al contrario de todas las políticas paternalistas históricas), sino que intenta ofrecer las condiciones necesarias para que el protegido pueda autoprotegerse en un futuro que sea el menos distante posible. Con respecto a la cuestión específica de la información y su manipulación, parece muy pertinente la sugerencia del "situacionista" Raoul Vaneighem, para quien "la libertad de expresión no será mas el sustituto de la libertad de acción cuando la vitalidad y la eficiencia que ella contiene llegaran a prevenir y a evitar las contrafacciones creando una adecuación entre la fraternidad de las palabras y la fraternidad de los hombres" (VAANEIGEM, 2003).

\section{Referências bibliográficas}

AGAMBEN, G. Homo sacer. Stanford (CA), Stanford University Press, 1996.

BARTHES, R. La retorica antica. Milano, Editore Bompiani, 1973.

DERRIDA, J. Voyous. Paris, Editions Galilée: 204-205, 2003.

KOTTOW, M. Introducción a la bioética. Santiago (Chile), Editorial Universitaria, 1995.

PRIETO, L. Pertinence et pratique. Paris, Editions de Minuit, 1975.

SCHRAMM, FR \& KOTTOW, M. Principios bioéticos en salud pública: limitaciones y propuestas, Cadernos de Saúde Pública, Rio de Janeiro,17(4): 949-956, 2001.

VAANEIGEM, R. Rien n'est sacré, tout peut se dire. Paris, La Découverte, : 23, 2003.

ŽIŽEK, S. Bem-vindo ao deserto do Real!: cinco ensaios sobre o 11 de setembro e datas relacionadas. São Paulo, Boitempo Editorial, 2003.

ŽIŽEK, S. O novo eixo da luta de classes, Folha de São Paulo. Mais! , 5 de septiembre: 8-11, 2004. 